\title{
Outcomes of Infected versus Symptomatic Sterile Walled-Off Pancreatic Necrosis Treated with a Minimally Invasive Therapy
}

\author{
Jong Jin Hyun ${ }^{1,2}$, Nadav Sahar ${ }^{1}$, Anand Singla ${ }^{3}$, Andrew S. Ross ${ }^{1}$, Shayan S. Irani ${ }^{1}$, S. Ian Gan ${ }^{1}$, Michael C. Larsen ${ }^{1}$, \\ Richard A. Kozarek ${ }^{1}$, and Michael Gluck ${ }^{1}$ \\ ${ }^{\prime}$ Digestive Disease Institute, Virginia Mason Medical Center, Seattle, WA, USA, ${ }^{2}$ Division of Gastroenterology and Hepatology, Korea University \\ College of Medicine, Seoul, Korea, and ${ }^{3}$ Division of Gastroenterology, Northwestern University, Chicago, IL, USA
}

\section{See editorial on page 135.}

Background/Aims: Acute pancreatitis complicated by walled-off necrosis (WON) is associated with high morbidity and mortality, and if infected, typically necessitates intervention. Clinical outcomes of infected WON have been described as poorer than those of symptomatic sterile WON. With the evolution of minimally invasive therapy, we sought to compare outcomes of infected to symptomatic sterile WON. Methods: We performed a retrospective cohort study examining patients who were undergoing dual-modality drainage as minimally invasive therapy for WON at a high-volume tertiary pancreatic center. The main outcome measures included mortality with a drain in place, length of hospital stay, admission to intensive care unit, and development of pancreatic fistulae. Results: Of the 211 patients in our analysis, 98 had infected WON. The overall mortality rate was $2.4 \%$. Patients with infected WON trended toward higher mortality although not statistically significant ( $4.1 \%$ vs $0.9 \%, p=0.19$ ). Patients with infected WON had longer length of hospitalization (29.8 days vs 17.3 days, $p<0.01$ ), and developed more spontaneous pancreatic fistulae $(23.5 \%$ vs $7.8 \%, p<0.01)$. Multivariate analysis showed that infected WON was associated with higher odds of spontaneous pancreatic fistula formation (odds ratio, 2.65; 95\% confidence interval, 1.20 to 5.85). Conclusions: This study confirms that infected WON has worse outcomes than sterile WON but also demonstrates that WON, once considered a significant cause of death, can be treated with good outcomes using minimally invasive therapy. (Gut Liver 2019;13:215-222)

Key Words: Pancreatitis, acute necrotizing; Infection; Mini- mally invasive therapy; Spontaneous pancreatic fistulae; Walled-off necrosis

\section{INTRODUCTION}

Necrotizing pancreatitis occurs in approximately 15\% of patients admitted to the hospital with acute pancreatitis, and walled-off necrosis (WON) develops as a sequela of acute necrotic collections. ${ }^{1-3}$ Complications of WON can be serious, with patients developing persistent organ failure that can lead to prolonged hospital stays, require treatment with multiple procedures, and even result in death. ${ }^{4,5}$ In a retrospective study of 99 patients with necrotizing pancreatitis, Perez et al. ${ }^{6}$ showed that 37\% of affected patients had infected WON and worse outcomes than were found in patients with sterile WON. In a 15year study of 167 patients with necrotizing pancreatitis who underwent surgery, Rodriguez et al. ${ }^{7}$ demonstrated that proven or assumed infection was the indication in 51\% of the cases. Moreover, among the subset of patient who underwent surgery for "persistent unwellness," infection was documented in 42\%, further indicating that outcomes and prognosis are worse in infected WON than in sterile WON. ${ }^{7}$ Therefore, intervention was advocated if WON became infected or symptomatic. Despite some series indicating the adequacy of conservative management, the development of infected WON is typically an indication for intervention. ${ }^{4,5}$

Historically, WON has been managed by open surgical debridement; however, given improvements and the increasing availability of procedural options involving minimally invasive therapy, such as video-assisted retroperitoneal debridement (VARD), direct endoscopic necrosectomy (DEN), and percutaneous drainage with or without endoscopic intervention, less-in-

Correspondence to: Michael Gluck

Digestive Disease Institute, Virginia Mason Medical Center, 1100 Ninth Avenue, Seattle, Washington 98101, USA

Tel: +1-206-223-2319, Fax: +1-206-341-1405, E-mail: Michael.Gluck@virginiamason.org

Received on May 25, 2018. Revised on August 23, 2018. Accepted on August 24, 2018. Published online February 12, 2019

pISSN 1976-2283 eISSN 2005-1212 https://doi.org/10.5009/gnl18234

@ This is an Open Access article distributed under the terms of the Creative Commons Attribution Non-Commercial License (http://creativecommons.org/licenses/by-nc/4.0) which permits unrestricted non-commercial use, distribution, and reproduction in any medium, provided the original work is properly cited. 
vasive techniques are currently preferred. ${ }^{8-14}$ While many studies have reported outcomes of various interventions for WON, few studies have specifically examined differences in clinical outcomes between infected versus sterile WON as treatments have gravitated toward more minimally invasive therapies. The aim of this study was to compare the clinical outcomes associated with symptomatic sterile versus infected WON in patients undergoing minimally invasive therapy at Virginia Mason Medical Center.

\section{MATERIALS AND METHODS}

\section{Patients}

The data related to all patients undergoing minimally invasive therapy for symptomatic or infected WON are maintained in a prospectively collected database approved by the Institutional
Review Board of Virginia Mason Medical Center (IRB08120). Informed consent was waived. The indications for managing WON are unrelenting pain, continued clinical deterioration, gastric/ duodenal/biliary obstruction, and recurrent fevers or persistent leukocytosis without other causes (Fig. 1). Among all patients who develop WON, our database was built to capture only those who undergo minimally invasive therapy, since approximately $70 \%$ of these patients are transferred to our hospital at some point in their disease and the disease process prior to referral is not always clear. In addition, patients who died during the initial stage of acute necrotizing pancreatitis and thus could not undergo minimally invasive therapy are also not included. From our database, we retrospectively reviewed the data obtained in patients who underwent combined percutaneous and endoscopic drainage (dual modality drainage, DMD) as a minimally invasive therapy between November 2007 and February 2017.

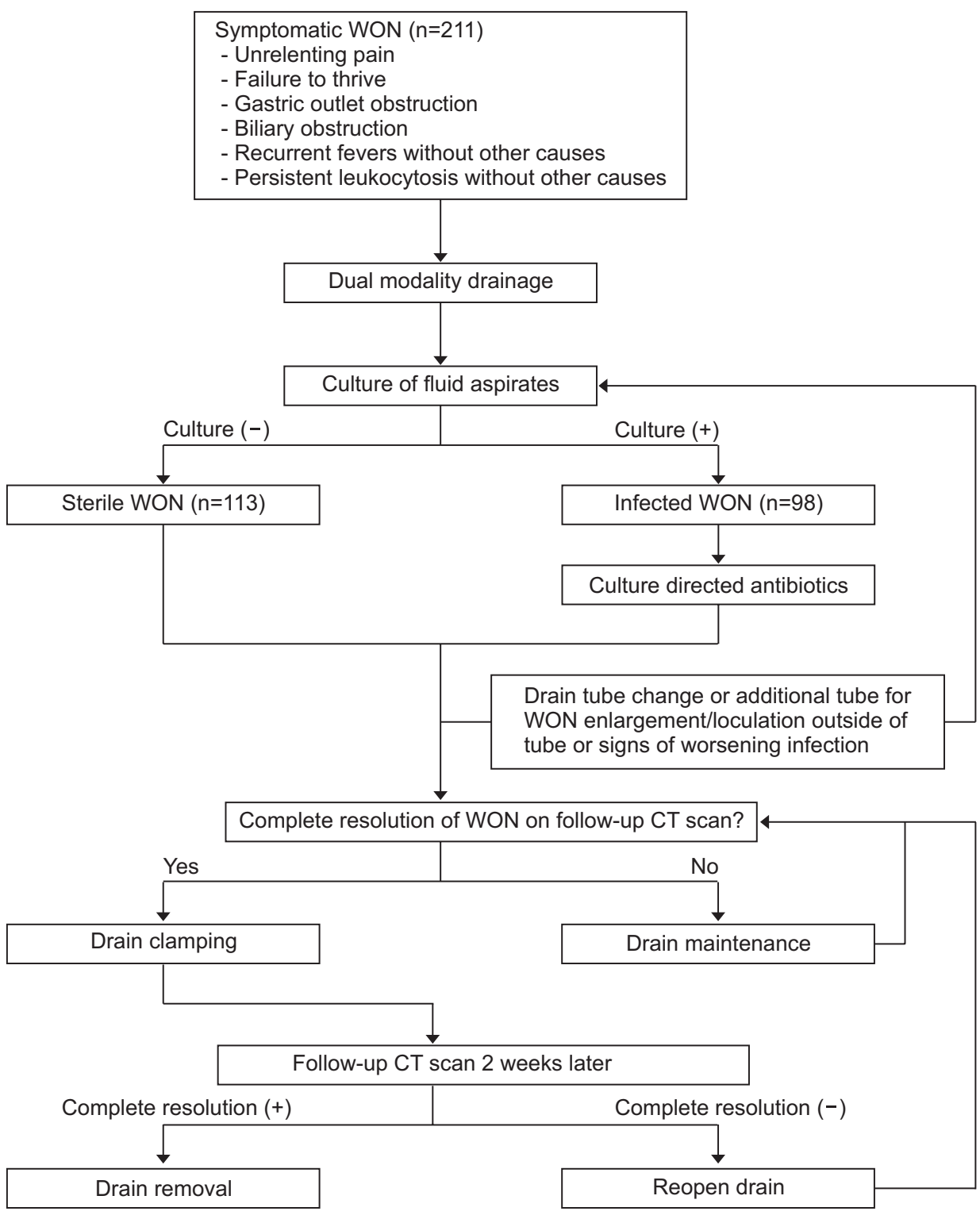

Fig. 1. Therapeutic flow diagram of patients who underwent minimally invasive therapy for walled-off pancreatic necrosis (WON). 
The database captures patient characteristics, including age at presentation, sex, and body mass index. Clinical information and factors related to necrotizing pancreatitis, including the most likely etiology of pancreatitis and the American Society of Anesthesiologists (ASA) classification, were obtained. When available, the size of fluid collection was determined using the largest reported dimension of the largest collection on crosssectional imaging along with the modified computed tomography severity index (CTSI). Symptomatic sterile collections were defined as those with gastric, duodenal, or biliary obstruction and those obtained in patients who did not progress clinically due to an inability to eat or failure to thrive. The following strict definition of infected necrosis was used: positive culture results from fluid aspirates obtained during initial placement of a percutaneous or endoscopic drain.

\section{Drainage techniques}

Over the past two decades, our institutional approach to the treatment of symptomatic and infected WON involving the use of minimally invasive therapy has evolved from percutaneous drainage alone to DMD, and this change has resulted in good clinical outcomes, as described previously. ${ }^{15-18}$ Briefly, in DMD (Fig. 2), a percutaneous drainage catheter is placed into the WON under computed tomography (CT) guidance after taking into consideration multiple factors, such as the location of fluid collection, the trajectory of the catheter, the ease of drainage, and accessibility for wound care. If the WON is considered to be accessible from the gastrointestinal tract (i.e., is located within 2 $\mathrm{cm}$ of the gastric or duodenal wall), the patient is then immediately transferred to an endoscopy suite for additional endoscopic drainage. The WON is entered either endoscopically with a needle-knife sphincterotome (Cook Endoscopy, Winston-Salem, NC, USA) or under endoscopic ultrasound (EUS) guidance us- ing a 19-gauge needle or EUS-directed transenteric drainage system (Navix; Boston Scientific, Natick, MA). After a guidewire is placed within the cavity, the tract is dilated, and two 7-Fr double pigtail stents or, more recently, lumen-apposing metal stents (LAMS) (AXIOS; Boston Scientific, Natick, MA, USA) are placed across the gastric or duodenal wall. After the procedure, percutaneous drains are flushed with 10 to $20 \mathrm{~mL}$ of saline 3 times a day. Although every attempt is made to postpone the drainage procedure until after 4 weeks from initiation of acute necrotizing pancreatitis, drainage is carried out within 4 weeks if the patient has unrelenting pain, develops recurrent fevers without other cause, or shows continued clinical deterioration despite intensive conservative management. Repeat percutaneous drain changes are performed for analogous reasons, that is, if the size of the WON increases, fever develops with no other cause, or the patient shows clinical deterioration (Fig. 1). Apart from DMD, endoscopic necrosectomy was not performed in any of the patients. When complete resolution of WON was documented on follow-up CT scan, the drains are clamped. If a follow-up CT scan taken 2 weeks later demonstrates an absence of residual fluid, the percutaneous drains are removed. A single dose of intravenous prophylactic antibiotics (cefazolin or levofloxacin) is administered prior to drainage. Afterwards, the choice of type and the duration of antibiotic use, if indicated, is tailored based on the culture results and after consultation with infectious disease specialists (Fig. 1).

\section{Outcomes}

The main outcome measures used to compare infected and symptomatic sterile WON were mortality with a drain in place (unresolved WON), the length of hospital stay, the use of critical care services (i.e., admission to an intensive care unit), and the development of spontaneous pancreatic fistulae. Development
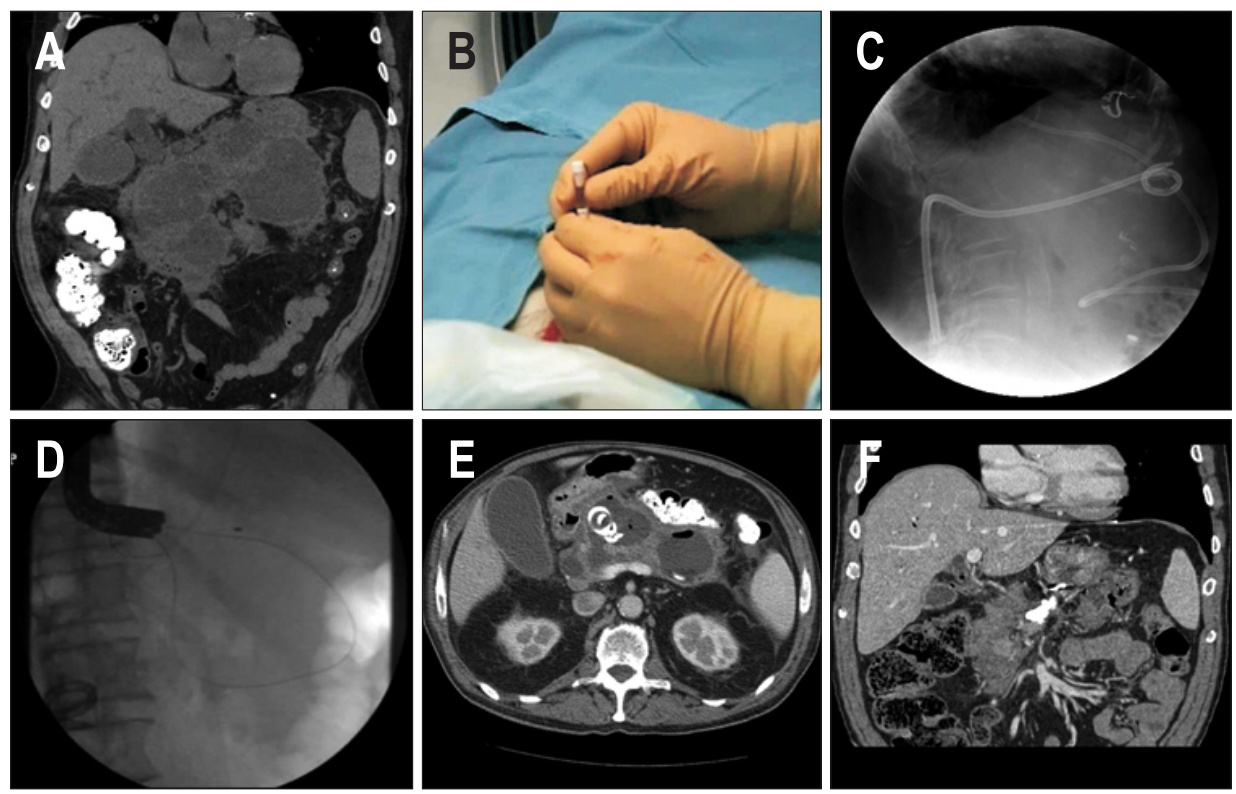

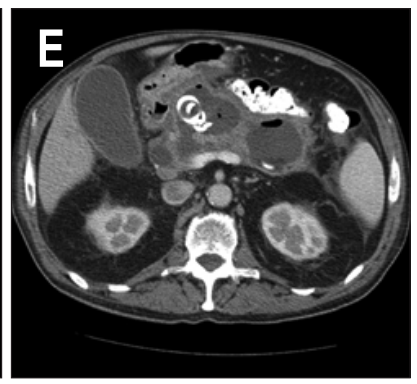

Fig. 2. Dual-modality drainage technique. (A) Walled-off necrosis (WON) is noted around the pancreas. (B) The area of WON was first accessed percutaneously for drainage under computed tomography (CT) guidance. (C) The percutaneous drainage tube is seen on a fluoroscopic image. (D) Drainage between the gastrointestinal tract and the area of WON was then performed endoscopically, and two double pigtail stents were inserted. (E) Followup CT scan taken 7 days after dualmodality drainage shows intact drainage tubes within the cyst, with a decrease in cyst size. (F) CT scan taken 4 months after the procedure demonstrates complete remission of WON. 
of spontaneous pancreatic fistulae was defined as the spontaneous formation of fistulae from the necrotic collection to an extrapancreatic location, that is, the biliary tract, gastrointestinal tract, pleural space, and peritoneum. For patients undergoing DMD, fistula formation in the stomach or duodenum was not considered spontaneous as this was the intended result of the endoscopic procedure unless the fistula was identified at a location different from the initial puncture site. For the same reason, fistula formation in the skin along the percutaneous tube tract was not considered spontaneous. Other outcomes assessed included the frequency of necessitating $>1$ percutaneous drainage, the interval between drain placement and removal, the number of total tube checks, the presence of disconnected duct syndrome, the occurrence of other complications (e.g., bleeding, gastric outlet obstruction, colonic obstruction, biliary stricture, and acute renal failure), and the number of total CT scans. The presence of disconnected duct syndrome was diagnosed by endoscopic retrograde cholangiopancreatography performed at the time of drainage or magnetic resonance cholangiopancreatography performed later in the course of treatment. ${ }^{16,17}$

\section{Statistical analysis}

Data were analyzed using IBM SPSS Statistics version 23.0 (IBM Corp., Armonk, NY, USA). Data are expressed as the mean \pm standard deviation or number $(\%)$ values. Comparative statistical analyses were performed using unpaired Student ttests for continuous variables and chi-square tests for categorical variables. $p$-values $<0.05$ were considered statistically significant. Multivariate logistic regression was used to assess the association between having infected WON and the occurrence of spontaneous pancreatic fistulae and other clinical parameters after controlling for confounding factors, such as patient characteristics, the etiology of pancreatitis and having multiple percutaneous drains.

\section{RESULTS}

\section{Baseline characteristics}

A total of 211 patients were included in this analysis. Of these, $98(46.4 \%)$ were classified as infected WON, and 113 (53.6\%) were classified as symptomatic sterile WON (Table 1). Infected WON patients were older at presentation than symptomatic sterile WON patients (mean age: 56.7 years vs 51.4 years, $\mathrm{p}=0.01$ ). Approximately two-thirds of the patients were male, and there was no difference in the sex ratio between the two groups. There was also no difference between the two groups with regards to etiologies of pancreatitis. The most common etiologies were gallstones $(52.1 \%)$, followed by alcohol

Table 1. Baseline Characteristics of Patients with Infected and Symptomatic Sterile WON

\begin{tabular}{|c|c|c|c|}
\hline Characteristic & Infected WON ( $\mathrm{n}=98)$ & Symptomatic sterile WON $(\mathrm{n}=113)$ & p-value \\
\hline Age, yr & $56.71 \pm 15.46$ & $51.35 \pm 15.76$ & 0.01 \\
\hline Female sex & $33(33.7)$ & $34(30.1)$ & 0.66 \\
\hline BMI, $\mathrm{kg} / \mathrm{m}^{2}$ & $31.20 \pm 6.34$ & $28.92 \pm 6.95$ & 0.01 \\
\hline Pancreatitis etiology & & & 0.58 \\
\hline Gallstones & $52(53.1)$ & $58(51.3)$ & \\
\hline Alcohol & 17 (17.3) & $26(23.0)$ & \\
\hline Triglycerides & $7(7.1)$ & $3(2.7)$ & \\
\hline Medications & $2(2.0)$ & 0 & \\
\hline Post-ERCP & $2(2.0)$ & $2(1.8)$ & \\
\hline Surgical/trauma & $3(3.1)$ & $4(3.5)$ & \\
\hline Ampullary adenoma & $1(1.0)$ & $2(1.8)$ & \\
\hline Pancreas divisum & $2(2.0)$ & $5(4.4)$ & \\
\hline Idiopathic & $12(12.2)$ & $13(11.5)$ & \\
\hline ASA classification & & & $<0.01$ \\
\hline I & $1(1.0)$ & 0 & \\
\hline II & $12(12.2)$ & 38 (33.9) & \\
\hline III & $63(64.3)$ & $62(55.4)$ & \\
\hline IV & $22(22.4)$ & $12(10.7)$ & \\
\hline Max dimension collection size, $\mathrm{cm}$ & $14.53 \pm 4.38$ & $14.19 \pm 4.54$ & 0.58 \\
\hline CTSI & $8.56 \pm 1.56$ & $7.72 \pm 1.63$ & $<0.01$ \\
\hline
\end{tabular}

Data are presented as mean \pm SD or number (\%).

WON, walled-off necrosis; BMI, body mass index; ERCP, endoscopic retrograde cholangiopancreatography; ASA, American Society of Anesthesiologists; CTSI, computed tomography severity index. 
(20.4\%). With regard for physical status, more patients had higher ASA grading scores in the infected WON group than in the symptomatic sterile WON group $(p<0.01)$. The maximum dimension of fluid collection size was equivalent between the patients with infected and symptomatic sterile WON. The CTSI was significantly higher in the infected WON group than in the symptomatic sterile WON group $(8.56 \pm 1.56$ vs $7.72 \pm 1.63$, $\mathrm{p}<0.01$ ).

\section{Clinical outcomes}

A comparison of the outcomes observed in infected WON and symptomatic sterile WON is summarized in Table 2. The overall mortality rate was very low, with only five patients (2.4\%) dying with the drain in place. The causes of death in these five patients were multiorgan failure $(n=1)$, recurrent gastrointestinal bleeding $(n=2)$, respiratory failure $(n=1)$, and heart failure $(n=1)$. Although the mortality rate was not significantly different between the two groups ( $p=0.19)$, it was arithmetically higher in the infected WON group $(4.1 \%, 4 / 98)$ than in the symptomatic sterile WON group (0.9\%, 1/113), indicating a clear trend toward worse outcomes in the infected WON group. Infected WON patients required longer hospital stays (29.8 days vs 17.0 days, $\mathrm{p}<0.01)$, more frequent use of critical care services $(37.8 \%$ vs $15.0 \%, \mathrm{p}<0.01$ ), and multiple percutaneous drain placement procedures $(31.6 \%$ vs $14.2 \%, \mathrm{p}<0.01)$, had a shorter time from the onset of acute pancreatitis to drain placement (58.0 days vs 98.7 days, $\mathrm{p}<0.01$ ), a longer time to drain removal (101.2 days vs 72.4 days, $p=0.01$ ), more tube checks ( 6.6 vs $5.2, p=0.01$ ), and a larger number of CT scans (8.9 vs 6.7, $\mathrm{p}<0.01$ ). Although there was no difference between infected WON and symptomatic sterile WON with regard for the presence of disconnected duct syndrome, those with infected WON more frequently developed spontaneous pancreatic fistulae ( $23.5 \%$ vs $7.8 \%, \mathrm{p}<0.01)$. After controlling for potential confounding factors, infected WON was also associated with higher odds of pancreatic fistula formation (odds ratio [OR], 2.494; 95\% confidence interval [CI], 1.031 to 6.033; $\mathrm{p}=0.043$ ), intensive care unit stay (OR, 2.649; 95\% CI, 1.201 to 5.846 ) and higher CTSI scores (OR, 1.363; 95\% CI, 1.108 to 1.677). With regard for other adverse events that occurred in patients who underwent DMD, the occurrence of bleeding $(n=15)$, gastric outlet obstruction $(n=12)$, colonic obstruction $(n=1)$, biliary stricture $(n=7)$, and acute renal failure $(n=13)$ were not different between the infected WON group and the symptomatic sterile WON group (Table 2).

\section{DISCUSSION}

In this high-volume, single-center, retrospective study, we

Table 2. Comparison of Clinical Outcomes between Infected and Symptomatic Sterile WON

\begin{tabular}{|c|c|c|c|}
\hline Clinical outcome & Infected WON (n=98) & Symptomatic sterile WON $(n=113)$ & p-value \\
\hline Mortality with drain in place & $4(4.1)$ & $1(0.9)$ & 0.19 \\
\hline Length of stay, day & $29.83 \pm 25.58$ & $17.02 \pm 16.60$ & $<0.01$ \\
\hline ICU stay required & 37 (37.8) & $17(15.0)$ & $<0.01$ \\
\hline$>1$ percutaneous drain & $31(31.6)$ & $16(14.2)$ & $<0.01$ \\
\hline Interval between pancreatitis onset and drain, day & $57.98 \pm 60.48$ & $98.67 \pm 119.88$ & $<0.01$ \\
\hline Interval between drain and removal, day & $101.18 \pm 102.58$ & $72.42 \pm 47.74$ & 0.01 \\
\hline No. of total tube checks & $6.60 \pm 3.86$ & $5.21 \pm 2.95$ & 0.01 \\
\hline Disconnected duct syndrome & $68(69.4)$ & $71(62.8)$ & 0.30 \\
\hline Spontaneous fistula & $23(23.5)$ & $9(7.8)$ & $<0.01$ \\
\hline Stomach & 1 & 0 & \\
\hline Small bowel & 5 & 2 & \\
\hline Colon & 11 & 4 & \\
\hline Pleura & 4 & 2 & \\
\hline Bile duct & 1 & 1 & \\
\hline Retroperitoneum & 1 & 0 & \\
\hline Bleeding & $6(6.1)$ & $9(8.0)$ & 0.60 \\
\hline Gastric outlet obstruction & $6(6.1)$ & $6(5.3)$ & 0.80 \\
\hline Colonic obstruction & $1(1.0)$ & 0 & 0.46 \\
\hline Biliary stricture & $3(3.1)$ & $4(3.5)$ & 1.00 \\
\hline Acute renal failure & $9(9.2)$ & $4(3.5)$ & 0.09 \\
\hline No. of total CT scans & $8.90 \pm 5.16$ & $6.73 \pm 3.42$ & $<0.01$ \\
\hline
\end{tabular}

Data are presented as number (\%) or the mean \pm SD.

WON, walled-off necrosis; ICU, intensive care unit; CT, computed tomography. 
examined clinical outcomes in treated WON, specifically with respect for the presence of infection. Several studies have demonstrated that mortality is higher in those with infected necrosis. However, previous studies have all had small sample sizes, and many did not directly compare infected WON with sterile WON. ${ }^{9,19-22}$ To our knowledge, our study is the largest study to compare clinical outcomes between infected and sterile WON in cases treated with minimally invasive therapy. The results of our study confirm that compared to sterile WON, infected WON predicts worse outcomes and showed a non-significant trend toward higher mortality $(4.1 \%$ vs $0.9 \%, \mathrm{p}=0.19)$. Surprisingly, overall mortality was very low (2.4\%) even when only infected WON patients were considered (4.1\%). This finding demonstrates that WON, which was once considered a highly morbid and significant cause of death, can be treated with good outcomes using minimally invasive therapy regardless of whether it is infected or sterile. The authors fully recognize that mortality is not the same between treated WON and severe acute pancreatitis (SAP) since a percentage of patients do not survive the inflammatory response and organ failure that occur during the initial stages of SAP. WON develops at a later stage, often when a patient may be stabilizing.

Currently, DEN and VARD are the most common procedures used to drain symptomatic WON. ${ }^{13}$ However, a variable subset of these patients require the addition of a percutaneous drain because of nonresolution, infection, or the extension of WON into areas not amenable to conventional drainage or endoscopic debridement. Although DMD is not a modality that is currently utilized as the standard of practice for treating WON at other pancreatic centers, the fact that the use of DMD led to single digit mortality implies that our minimally invasive therapy is as effective as and representative of minimally invasive therapy for WON. At our institution, either two 7-Fr double pigtail stents or a LAMS are placed across the gastric or duodenal wall during DMD. Although LAMS have recently been inserted at a higher rate, when we compared the two endoscopic transgastric stent techniques, we found no differences between the treatment outcomes for WON. ${ }^{23}$ Since the implementation of DMD for the treatment of WON at our institution, no patient has required open surgical necrosectomy, undergone VARD or needed a distal pancreatectomy for a persistent percutaneous fistula, ${ }^{16,18}$ a situation common in previous series in patients treated with percutaneous drainage alone. ${ }^{24,25}$ Nevertheless, our study fails to define the total mortality in SAP since only a select group of patients survived the initial severe disease, presented with WON, and then underwent DMD were included in the analysis. Patients who presented with organ failure and died early during the course of illness were not included. This could be one of the reasons for the low mortality of $2.4 \%$ observed in the current study, which was lower than that reported in a recently published multicenter randomized trial that found a mortality rate of $18 \%$ in patients with infected collections that were treated endoscopically, even in those with a median Sequential Organ Failure Assessment score of $0 .{ }^{26}$ As most of the patients in this series were transferred from outside institutions, obtaining true mortality figures would require researchers to determine the number of patients admitted with acute pancreatitis and the concomitant mortality rates across multiple centers with variable expertise and resources for treating such patients.

In addition to requiring longer hospital stays and more critical care services and showing a trend toward higher mortality, patients with infected WON also more frequently developed spontaneous pancreatic fistulae. Even after adjusting for patient characteristics, the etiology of pancreatitis and the presence of multiple percutaneous drains, infected WON patients had 3-fold higher odds of spontaneous pancreatic fistulae formation. The reason for this association remains unclear. Spontaneous pancreatic fistulae can result from severe necrotizing pancreatitis as well as interventional pancreatic procedures. Since all patients in our study underwent placement of a percutaneous drain and endoscopic drainage, it is not clear whether procedural manipulation of pancreatic fluid collection contributed to the occurrence of spontaneous pancreatic fistulae in structures other than the skin and the stomach or duodenum. Alternatively, infection of the necrotic collection could lead to increased inflammation of contiguous structures, resulting in damage to the surrounding structures and spontaneous fistulae formation. It is also possible that infection of the fluid collection results from spontaneous pancreatic fistulae developing in a nonsterile structure, such as the colon, leading to bacterial colonization of the fluid collection. In a prior study, Ho and Frey ${ }^{27}$ found that 25 out of $136(18 \%)$ patients with SAP developed spontaneous pancreatic fistulae. However, the rate was much higher in patients who underwent surgical treatment for necrotizing pancreatitis. ${ }^{28}$ For patients undergoing pancreatic resection for any reason, the incidence of pancreatic fistulae ranged between 9.9\% and $28.5 \%$. $^{29}$

Infected WON, in our patient population, was an indication for intervention. It should be noted that there is some evidence suggesting that prompt intervention may not always be necessary in infected WON. Some authors have advocated that conservative management with antibiotics and careful monitoring may be adequate. ${ }^{30,31}$ However, this could increase the incidence of multidrug-resistant organisms and Clostridium difficile infection, and care should be taken to avoid the overuse of antibiotics. $^{32,33}$ The subset of patients who seem most appropriate for noninvasive management are clinically stable patients, a group not found in our series as the vast majority were transferred to our hospital due to clinical instability. Recent guidelines suggest that patients with sterile WON can appear to be as clinically ill as those with infected WON; thus, symptomatic sterile WON is also drained.

This study is not without its limitations. Our data represent the experience of a single high-volume, tertiary referral center 
for patients with complex pancreaticobiliary disorders. Given the availability of advanced endoscopists and experienced interventional radiologists, our results may not apply to lower volume centers that do not have the same dedicated resources or expertise in treating pancreatic diseases. Analyses of large, administrative datasets have shown that a higher hospital volume of acute pancreatitis is associated with better clinical outcomes. ${ }^{34}$ In addition, the patients in the current study had fluid collections that could be reached endoscopically, and these data therefore cannot be applied to WON that occurs more than $2 \mathrm{~cm}$ from the gastric or duodenal wall on CT scan. Our cohort does not reflect the potential differences in outcomes that may occur between patients with infected and sterile WON located away from the lumen of the gastrointestinal tract; however, these are infrequently found in clinical practice. Therefore, our conclusions appear valid for the subset of patients with WON in whom combined percutaneous and endoscopic drainage is feasible.

In conclusion, infected WON patients have longer length of stay, a higher use of critical care services, and a higher incidence of spontaneous fistula than were found in symptomatic sterile WON. Although a longer, more complicated hospital course can be anticipated in patients with infected WON, providers can nevertheless expect good outcomes in this era of minimally invasive therapy.

\section{CONFLICTS OF INTEREST}

No potential conflict of interest relevant to this article was reported.

\section{ACKNOWLEDGEMENTS}

The authors thank Terri Davis Smith for administrative, technical, and material support.

\section{REFERENCES}

1. Banks PA, Freeman ML; Practice Parameters Committee of the American College of Gastroenterology: practice guidelines in acute pancreatitis. Am J Gastroenterol 2006;101:2379-2400.

2. Banks PA, Bollen TL, Dervenis C, et al. Classification of acute pancreatitis-2012: revision of the Atlanta classification and definitions by international consensus. Gut 2013;62:102-111.

3. Zhao K, Adam SZ, Keswani RN, Horowitz JM, Miller FH. Acute pancreatitis: revised Atlanta classification and the role of crosssectional imaging. AJR AM J Roentgenol 2015;205:W32-W41.

4. Tenner S, Baillie J, DeWitt J, Vege SS; American College of Gastroenterology. American College of Gastroenterology guideline: management of acute pancreatitis. Am J Gastroenterol 2013;108:1400-1415.

5. Working Group IAP/APA Acute Pancreatitis Guidelines. IAP/APA evidence-based guidelines for the management of acute pancreati- tis. Pancreatology 2013;13:e1-e15.

6. Perez A, Whang EE, Brooks DC, et al. Is severity of necrotizing pancreatitis increased in extended necrosis and infected necrosis? Pancreas 2002;25:229-233.

7. Rodriguez JR, Razo A0, Targarona J, et al. Debridement and closed packing for sterile or infected necrotizing pancreatitis: insights into indications and outcomes in 167 patients. Ann Surg 2008;247:294-299.

8. Clancy TE, Ashley SW. Current management of necrotizing pancreatitis. Adv Surg 2002;36:103-121.

9. Besselink MG, Verwer TJ, Schoenmaeckers EJ, et al. Timing of surgical intervention in necrotizing pancreatitis. Arch Surg 2007;142:1194-1201.

10. Besselink MG, van Santvoort HC, Nieuwenhuijs VB, et al. Minimally invasive 'step-up approach' versus maximal necrosectomy in patients with acute necrotizing pancreatitis (PANTER trial): design and rationale of a randomised controlled multicenter trial [ISRCTN13975868]. BMC Surg 2006;6:6.

11. van Santvoort HC, Bakker OJ, Bollen TL, et al. A conservative and minimally invasive approach to necrotizing pancreatitis improves outcome. Gastroenterology 2011;141:1254-1263.

12. Gardner TB, Chahal P, Papachristou GI, et al. A comparison of direct endoscopic necrosectomy with transmural endoscopic drainage for the treatment of walled-off pancreatic necrosis. Gastrointest Endosc 2009;69:1085-1094.

13. Boumitri C, Brown E, Kahaleh M. Necrotizing pancreatitis: current management and therapies. Clin Endosc 2017;50:357-365.

14. Kawakami H, Itoi T, Sakamoto N. Endoscopic ultrasound-guided transluminal drainage for peripancreatic fluid collections: where are we now? Gut Liver 2014;8:341-355.

15. Gluck M, Ross A, Irani S, et al. Endoscopic and percutaneous drainage of symptomatic walled-off pancreatic necrosis reduces hospital stay and radiographic resources. Clin Gastroenterol Hepatol 2010;8:1083-1088.

16. Ross A, Gluck M, Irani S, et al. Combined endoscopic and percutaneous drainage of organized pancreatic necrosis. Gastrointest Endosc 2010;71:79-84.

17. Gluck M, Ross A, Irani S, et al. Dual modality drainage for symptomatic walled-off pancreatic necrosis reduces length of hospitalization, radiological procedures, and number of endoscopies compared to standard percutaneous drainage. J Gastrointest Surg 2012;16:248-256

18. Ross AS, Irani S, Gan SI, et al. Dual-modality drainage of infected and symptomatic walled-off pancreatic necrosis: long-term clinical outcomes. Gastrointest Endosc 2014;79:929-935.

19. Beger HG, Rau B, Isenmann R. Natural history of necrotizing pancreatitis. Pancreatology 2003;3:93-101.

20. Beger HG, Bittner R, Block S, Büchler M. Bacterial contamination of pancreatic necrosis. A prospective clinical study. Gastroenterology 1986;91:433-438.

21. Petrov MS, Kukosh MV, Emelyanov NV. A randomized controlled trial of enteral versus parenteral feeding in patients with predicted 
severe acute pancreatitis shows a significant reduction in mortality and in infected pancreatic complications with total enteral nutrition. Dig Surg 2006;23:336-344.

22. Bhansali SK, Shah SC, Desai SB, Sunawala JD. Infected necrosis complicating acute pancreatitis: experience with 131 cases. Indian J Gastroenterol 2003;22:7-10.

23. Sahar N, Kozarek R, Kanji ZS, et al. Do lumen-apposing metal stents (LAMS) improve treatment outcomes of walled-off pancreatic necrosis over plastic stents using dual-modality drainage? Endosc Int Open 2017;5:E1052-E1059.

24. Freeny PC, Hauptmann E, Althaus SJ, Traverso LW, Sinanan M. Percutaneous CT-guided catheter drainage of infected acute necrotizing pancreatitis: techniques and results. AJR Am J Roentgenol 1998;170:969-975.

25. Traverso LW, Kozarek RA. Interventional management of peripancreatic fluid collections. Surg Clin North Am 1999;79:745-757.

26. van Brunschot S, van Grinsven J, van Santvoort HC, et al. Endoscopic or surgical step-up approach for infected necrotising pancreatitis: a multicenter randomised trial. Lancet 2018;391:51-58.

27. Ho HS, Frey CF. Gastrointestinal and pancreatic complications associated with severe pancreatitis. Arch Surg 1995;130:817-822.

28. Tsiotos GG, Smith CD, Sarr MG. Incidence and management of pancreatic and enteric fistulas after surgical management of severe necrotizing pancreatitis. Arch Surg 1995;130:48-52.

29. Bassi C, Butturini G, Molinari E, et al. Pancreatic fistula rate after pancreatic resection: the importance of definitions. Dig Surg 2004;21:54-59.

30. Garg PK, Sharma M, Madan K, Sahni P, Banerjee D, Goyal R. Primary conservative treatment results in mortality comparable to surgery in patients with infected pancreatic necrosis. Clin Gastroenterol Hepatol 2010;8:1089-1094.

31. Mouli VP, Sreenivas V, Garg PK. Efficacy of conservative treatment, without necrosectomy, for infected pancreatic necrosis: a systematic review and meta-analysis. Gastroenterology 2013;144:333-340.

32. Barnes SL, Rock C, Harris AD, Cosgrove SE, Morgan DJ, Thom KA. The impact of reducing antibiotics on the transmission of multidrug-resistant organisms. Infect Control Hosp Epidemiol 2017;38:663-669.

33. Trikudanathan G, Munigala S. Impact of Clostridium difficile infection in patients hospitalized with acute pancreatitis: a population based cohort study. Pancreatology 2017;17:201-202.

34. Singla A, Simons J, Li Y, et al. Admission volume determines outcome for patients with acute pancreatitis. Gastroenterology 2009;137:1995-2001. 